\title{
Erratum: Contribution of nitrogen atoms and ions to the luminescence emission during femotosecond filamentation in air [Phys. Rev. A 93, 013405 (2016)]
}

\author{
Su-Yu Li, Shu-Chang Li, Lai-Zhi Sui, Yuan-Fei Jiang, An-Min Chen, and Ming-Xing Jin \\ (Received 29 June 2016; published 7 November 2016)
}

DOI: 10.1103/PhysRevA.94.059901

In our recent work, we have investigated the influence of laser polarization state on the luminescence emission during femtosecond filamentation in air and reported on a significant difference between the emission behavior of the 391-nm line and the other ones under different pump laser polarizations. We attributed this difference to the contribution of the atomic ions to the luminescence emission around $391 \mathrm{~nm}$. However, when we revisited this phenomenon recently, we found that a mistake was made in the original article, which greatly affected our analysis as well as our conclusion. What is more, as was pointed out by Ilyin, there should be no contribution of $\mathrm{N}^{+}$ lines to the luminescence emission from filaments around the 391-nm line [1]. In the original article, the spectra are measured by the spectrometer (SpectraPro 500i) with 150 grooves $/ \mathrm{mm}$ grating; it is simply to obtain $0.7 \mathrm{~nm}$ spectral resolution. As a result, the 426.8-nm line [coming from $C^{3} \Pi_{u}(v=$ 1) $-B^{3} \Pi_{g}\left(v^{\prime}=5\right)$ transition] and the 427.5-nm one [coming from $B^{2} \Sigma_{u}^{+}(v=0)-X^{2} \Sigma_{g}^{+}\left(v^{\prime}=1\right)$ transition] overlap with each other and both of them contribute to the 428-nm line emission. However, when we use the spectrometer (SpectraPro 500i) with 1200 grooves $/ \mathrm{mm}$ grating (about $0.1 \mathrm{~nm}$ spectral resolution) to conduct the experiment again, the two lines can be clearly distinguished, as shown in Fig. 1. In the original article, we regarded the 426.8-nm line as the 427.5-nm one, i.e., the 428-nm line. The former comes from the nitrogen molecule $\mathrm{N}_{2}$ while the latter comes from nitrogen molecular ion $\mathrm{N}_{2}{ }^{+}$, therefore their emission behaviors under different laser polarizations should be different. Here, we name the 426.8-nm line as the "false 428-nm line" and the 427.5-nm line as the "real 428-nm line."

Two effects play important roles in the relative intensity between the luminescence from nitrogen molecule $\mathrm{N}_{2}$ and nitrogen molecular ion $\mathrm{N}_{2}^{+}$: one is the higher ionization efficiency of linearly polarized laser pulses, and the other one is the impact excitation resulting from electrons with higher kinetic energy which are generated by circularly polarized laser pulses [2]. In the case of circular laser polarization, the maxima of the electron energy distribution function shift to larger values [3]; in other words, the average electron energy is larger in the case of circular polarization, making the population of $\mathrm{N}_{2}\left(C^{3} \Pi_{u}\right)$ and $\mathrm{N}_{2}{ }^{+}\left(B^{2} \Sigma_{u}^{+}\right)$levels larger. It can be seen from dashed blue curves in Figs. 1(a)-1(d) that the false 428-nm line is stronger than the real 428-nm line, and the former is much stronger than the latter in the longer focal length case [dashed blue curves in Figs. 1(c) and 1(d)], which can be attributed to the fact that the cross section of the impact excitation for $\mathrm{N}_{2}$ is larger than that for $\mathrm{N}_{2}{ }^{+}$when the electrons share the same kinetic energy [4]. In contrast, in the case of linear laser polarization, especially in the shorter focal length case, due to the higher ionization efficiency of linearly polarized laser pulse, more plasmas are generated, making the luminescence from $\mathrm{N}_{2}{ }^{+}$stronger, therefore, the real 428-nm line is is much stronger than the false 428-nm line, as shown by the solid red curves in Figs. 1(a) and 1(b), while in the longer focal length case, the ionization effect becomes weak and thus fewer $\mathrm{N}_{2}{ }^{+}$are generated; as a result, the false 428-nm line is comparable with the real 428-nm line in intensity and even becomes more intense than the real 428-nm line when the pulse energy is $0.8 \mathrm{~mJ}$ and focal length is $100 \mathrm{~cm}$ [solid red curve in Fig. 1(c)]. In our work as well as elsewhere [3], the peak value of each spectral line is taken as its intensity. Since the false $428-\mathrm{nm}$ line is more intense than the real 428-nm line, we took the false 428-nm line as the 428-nm line throughout the original paper, leading to the incorrect results and conclusion in the original article. The above results are obtained in the case that the gate width of ICCD is 3 ns. It is well known that $\mathrm{N}_{2}{ }^{+}$emission lifetime is about 100 ps $[5,6]$ and $\mathrm{N}_{2}$ emission lifetime is several hundred ps, and therefore selection of the gate width of ICCD will influence the intensity ratio $I(427.5 \mathrm{~nm}) / I(426.8 \mathrm{~nm})$. If the gate width was narrower, say $0.5 \mathrm{~ns}$, the intensity of the $427.5-\mathrm{nm}$ line is much higher than that of the 426.8-nm line in most cases. Under this circumstance, the 426.8-nm line would not be regarded as the

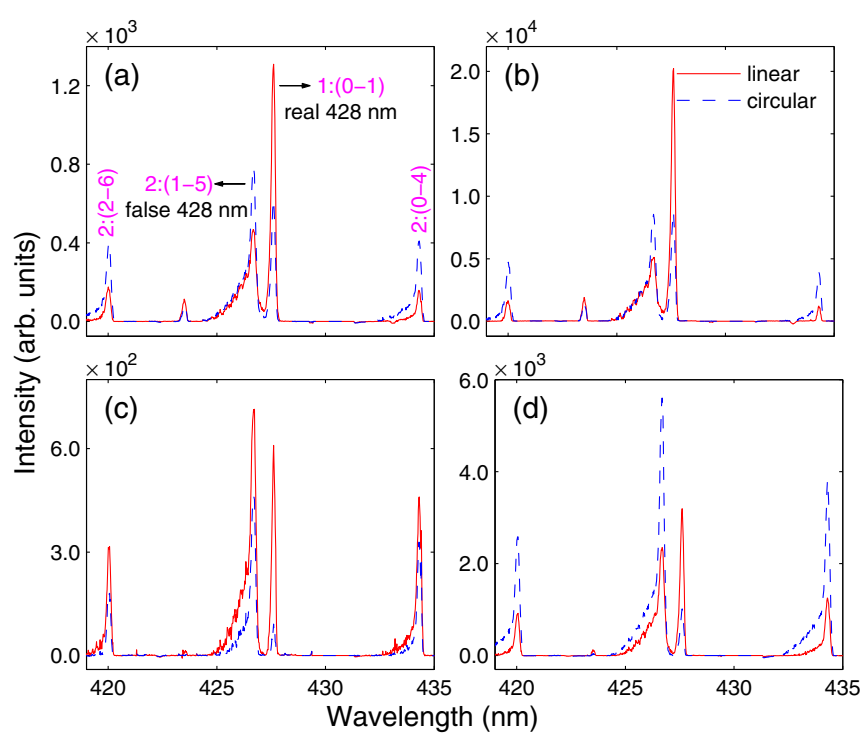

FIG. 1. Spectra emitted from the filament generated by the linearly (solid red curves) and circularly (dashed blue curves) polarized pulses as the laser energy is (a) 0.8 and (b) $2.0 \mathrm{~mJ}$ when the focal length is $40 \mathrm{~cm}$; the laser energy is (c) 0.8 and (d) $2.0 \mathrm{~mJ}$ when the focal length is $100 \mathrm{~cm}$. The spectra are measured by the spectrometer (SpectraPro 500i) with 1200 grooves/mm grating. 


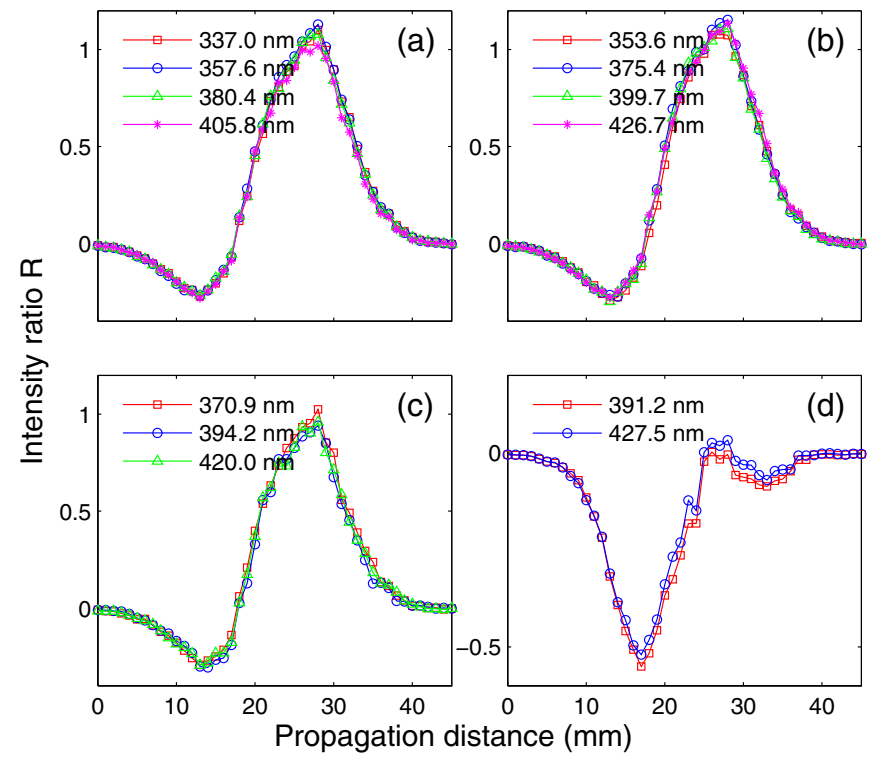

FIG. 2. Intensity ratios $R$ of (a) 337.0-, 357.6-, 380.4-, and 405.8-nm lines; (b) 353.6-, 375.4-, 399.7-, and 426.7-nm lines; (c) 370.9-, 394.2-, and 420-nm lines; and (d) 391.2- and 427.5-nm lines as the incident energy is $3.1 \mathrm{~mJ}$ and the focal length is $40 \mathrm{~cm}$. The spectra are measured by the spectrometer (SpectraPro 500i) with 1200 grooves/mm grating.

428-nm one, and thus the incorrect results and conclusion in the original article can be avoided.

Here, we present the intensity ratios $R\left(R=\left[I_{\text {circular }}(z)-\right.\right.$ $\left.\left.I_{\text {linear }}(z)\right] / I_{\text {linear,max }}\right)$ of 337.0-, 357.6-, 380.4-, and 405.8nm lines [coming from $C^{3} \Pi_{u}(v=0)-B^{3} \Pi_{g}\left(v^{\prime}=0,1,2,3\right)$ transition] in Fig. 2(a), those of 353.6-, 375.4-, 399.7-, and

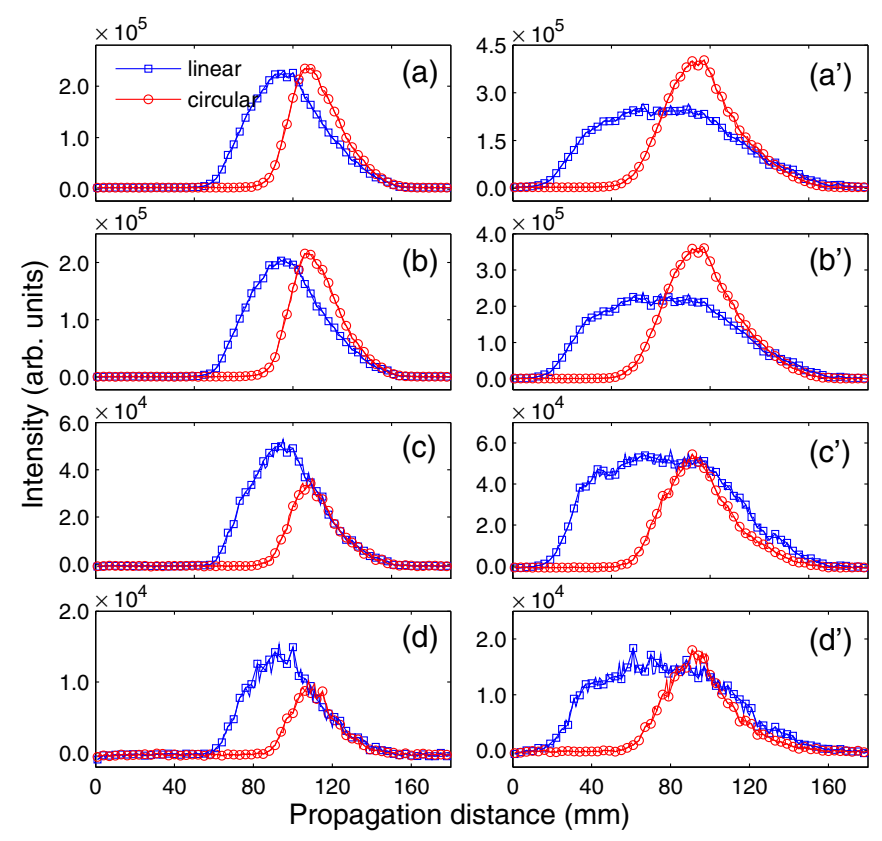

FIG. 3. Variation of the intensity of (a), $\left(a^{\prime}\right) 337-,(b),\left(b^{\prime}\right) 380-$, (c), (c') 391-, and (d), (d') 428-nm spectral lines along the propagation axis. The focal length is $100 \mathrm{~cm}$, and the pulse energy is (a)-(d) $2.0 \mathrm{~mJ}$ and $\left(\mathrm{a}^{\prime}\right)-\left(\mathrm{d}^{\prime}\right) 3.1 \mathrm{~mJ}$.

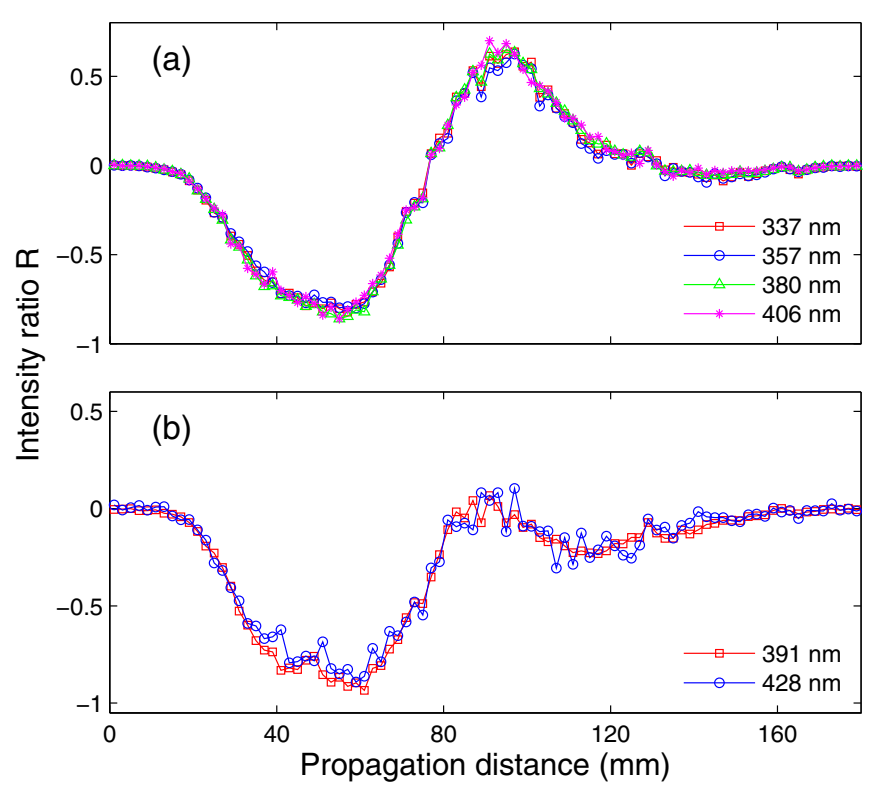

FIG. 4. Intensity ratios $R$ of (a) 337-, 357-, 380-, and 406-nm lines and (b) 391- and 428-nm lines as the incident energy is $3.1 \mathrm{~mJ}$ and the focal length is $100 \mathrm{~cm}$.

426.7-nm lines [coming from $C^{3} \Pi_{u}(v=1)-B^{3} \Pi_{g}\left(v^{\prime}=\right.$ $2,3,4,5)$ transition] in Fig. 2(b), those of 370.9-, 394.2-, and 420-nm lines [coming from $C^{3} \Pi_{u}(v=2)-B^{3} \Pi_{g}\left(v^{\prime}=\right.$ $4,5,6)$ transition] in Fig. 2(c), and those of 391.2- and 427.5$\mathrm{nm}$ lines [coming from $B^{2} \sum_{u}^{+}(v=0)-X^{2} \sum_{g}^{+}\left(v^{\prime}=0,1\right)$ transition] in Fig. 2(d). It can be seen from the figure that the intensity ratio of the real 428-nm line is identical to that of the 391-nm line [see Fig. 2(d)], and those of spectral lines from $\mathrm{N}_{2}$ are consistent with each other, the false 428-nm line included [see Figs. 2(a)-2(c)].
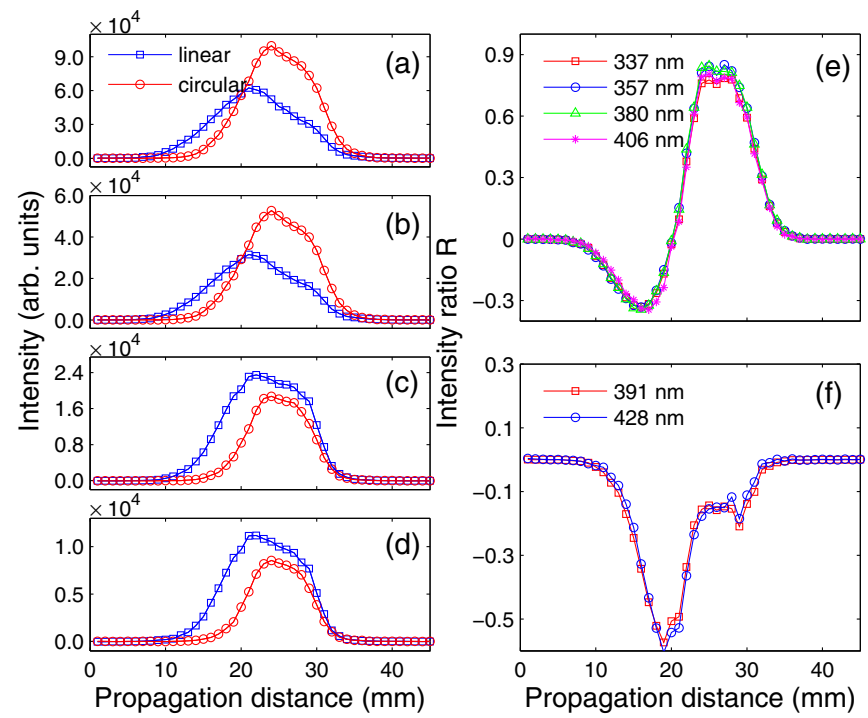

FIG. 5. Variation of the intensity of (a) 337-, (b) 380-, (c) 391-, and (d) 428-nm spectral lines along the propagation axis. (e),(f) Intensity ratio $R$ of different spectral lines. The focal length is $40 \mathrm{~cm}$, and the pulse energy is $3.1 \mathrm{~mJ}$. 
As has been stated above, there is no difference between the emission behaviors of 391- and 428-nm lines under different polarizations, that is to say, the emission mechanism of 391- and 428-nm lines is the same. In combination with the convincing and detailed analysis of Ilyin [1], we can say that there is no contribution of nitrogen atoms and ions to the nitrogen luminescence emission during femtosecond filamentation in air.

The results presented in Figs. 3-5 in the original article are the false 428-nm line, which leads to the incorrect result that there exists a great difference between the 391- and 428-nm spectral lines under different polarizations. If we replace it with the real 428-nm line, the results are completely different: the emission behaviors of the 391- and 428-nm lines are consistent with each other, similar to the case of the spectral lines from $\mathrm{N}_{2}$. Figures $3-5$ in the original article should be replaced by Figs. 3-5 in this erratum.

In addition, in Table $I$, the amplification ratios $I_{\mathrm{s}} / I_{1}$ (linear) and $I_{\mathrm{s}}^{\prime} / I_{1}^{\prime}$ (circular) for the 428-nm line should be 9.14 and 7.06, which is almost identical to those of the 391-nm line.

We apologize for our carelessness and nonrigorousness. We would like to deeply and sincerely thank A. A. Ilyin for analyzing the phenomena and alerting us about the mistake in the original article.
[1] A. A. Ilyin (private communication).

[2] Y. Shi, A. Chen, Y. Jiang, S. Li, and M. Jin, Opt. Commun. 367, 174 (2016).

[3] S. Mitryukovskiy, Y. Liu, P. Ding, A. Houard, A. Couairon, and A. Mysyrowicz, Phys. Rev. Lett. 114, 063003 (2015).
[4] Y. Itikawa, J. Phys. Chem. Ref. Data 35, 31 (2006).

[5] F. Martin, R. Mawassi, F. Vidal, I. Gallimberti, D. Comtois, H. Pépin, J. C. Kieffer, and H. P. Mercure, Appl. Spectrosc. 56, 1444 (2002).

[6] A. A. Ilyin, S. S. Golik, and K. A. Shmirko, Spectrochim. Acta Part B 112, 16 (2015). 